\title{
Microstructure and Bend Ductility of W-0.3 mass\% TiC Alloys Fabricated by Advanced Powder-Metallurgical Processing
}

\author{
Yasuhiro Ishijima $^{1, * 1}$, Hiroaki Kurishita ${ }^{1, * 2}$, Hideo Arakawa ${ }^{1}$, Masayuki Hasegawa ${ }^{1}$, \\ Yutaka Hiraoka ${ }^{2}$, Tomohiro Takida ${ }^{3}$ and Katsushi Takebe ${ }^{3}$ \\ ${ }^{1}$ International Research Center for Nuclear Materials Science, IMR, Tohoku University, Oarai, Ibaraki 311-1313, Japan \\ ${ }^{2}$ Okayama University of Science, Ridai-cho, Okayama 700-0005, Japan \\ ${ }^{3}$ Allied Tech Inc., Iwase-koshi-machi, Toyama 931-8543, Japan
}

\begin{abstract}
Low temperature embrittlement, recrystallization embrittlement and radiation embrittlement in tungsten and its alloys are critical issues for use as high heat flux components and high-power density structural materials. In order to establish a process for microstructural control to improve the resistance to such embrittlement, modification of powder-metallurgical processing is proposed to avoid three microstructural factors giving detrimental effects on the ductility: (1) precipitation of the brittle $\mathrm{W}_{2} \mathrm{C}$ phase, (2) heterogeneity in grain size and particle distributions, and (3) loss of carbon which is a constituent of transition metal carbides. The processing was applied to fabricate $\mathrm{W}-0.3 \mathrm{mass} \% \mathrm{TiC}$ alloys with a microstructure of fine grains and nano-sized dispersoids of TiC. Transmission electron microstructural observations and three-point bending tests at room temperature were performed on the alloys in the unirradiated state. It is demonstrated that the developed alloys are almost free from the three microstructural factors and exhibit appreciable room-temperature ductility before fracture in the as-forged and as-rolled states, but not in the as-HIPed state. This beneficial effect of plastic working on ductility improvement is strongly dependent on grain size and becomes prominent with decreasing grain size. Success in fabricating consolidated bodies with grain sizes as small as $0.17 \sim 0.4 \mu \mathrm{m}$ and a high relative density of around $99 \%$ is presented.
\end{abstract}

(Received November 1, 2004; Accepted January 31, 2005)

Keywords: tungsten, embrittlement, TiC dispersoid, fine grains, ductility, processing, mechanical alloying, plastic working, relative density

\section{Introduction}

Tungsten (W) is superior to other materials in many respects, including high melting point, low thermal expansion coefficient, low sputtering yields, low tritium inventory and excellent compatibility with liquid metals. ${ }^{1)}$ Therefore, $\mathrm{W}$ and its alloys are very promising for use as high heat flux components and high-power density structural materials in radiation environments. However, they are known to exhibit serious embrttilement in several regimes, i.e., low temperature embrittlement, recrystallization embrittlement and radiation embrittlement. These types of embrittlement are microstructure-sensitive and one of the authors has believed that the most effective microstructure to alleviate such embrittlement consists of fine grains and finely dispersed nano-particles of transition metal carbides. Such microstructural refinement can be achieved by materials processing based on powder metallurgical $(\mathrm{P} / \mathrm{M})$ methods including mechanical alloying (MA) ${ }^{2}$ and hot isostatic pressing (HIP) under a controlled atmosphere with negligible amounts of oxygen and nitrogen. Therefore, the authors have been trying to establish a process for microstructural refinement in at first Mo, ${ }^{3-8)}$ and then W. ${ }^{6-9-12)}$ They conducted mechanical alloying (MA) of powders of $\mathrm{W}, \mathrm{TiC}$ or $\mathrm{Ti}$ and $\mathrm{C}$, sintering of the MA treated powder by vacuum hot pressing (VHP) and hot isostatic pressing (HIP), followed by hot forging and hot rolling. The developed alloys exhibited improved ductility and high recrystallization temperature, but considerable difference was seen among the alloys. ${ }^{11)}$ The difference

\footnotetext{
${ }^{* 1}$ Graduate Student, Tohoku University, Oarai, Ibaraki 311-1313, Japan. Present address: Japan Atomic Energy Research Institute, Tokai-Mura, Ibaraki 319-1195, Japan

${ }^{* 2}$ Corresponding author, E-mail: kurishi@imr.tohoku.ac.jp
}

was considered to be attributable mainly to three microstructural factors giving detrimental effects on the ductility and recrystallization temperature; (1) precipitation of the brittle $\mathrm{W}_{2} \mathrm{C}$ phase, (2) heterogeneity in grain size and particle distributions and (3) significant loss of carbon necessary for the formation of finely dispersed particles of transition metal carbides. These three factors must be eliminated for practical use of $\mathrm{W}$ alloys.

In this paper, a process to avoid the three factors is proposed and applied to fabricate $\mathrm{W}-0.3$ mass $\% \mathrm{TiC}$ alloys with improved resistance to the embrittlement. Microstructures and mechanical properties of the alloys in the unirradiated state are examined. It is shown that the alloys have microstructures almost free from the three factors. Ductility improvement is achieved by plastic working after consolidation and can be remarkably enhanced by further grain refinement. Achieved grain sizes as small as $0.17 \sim$ $0.4 \mu \mathrm{m}$ in consolidated bodies is presented.

\section{Improved Processes for Solving the Problems}

In the following, the cause of each of the above three microstructural factors is discussed and the process to avoid each of the factors is presented.

\subsection{Precipitation of the brittle $\mathrm{W}_{2} \mathrm{C}$ phase}

The $\mathrm{W}_{2} \mathrm{C}$ phase is known to be very brittle. Our previous studies showed that the brittle $\mathrm{W}_{2} \mathrm{C}$ phase formed even without adding carbon or its compounds to $\mathrm{W}$ and had no orientation relationship with a $\mathrm{W}$ matrix. ${ }^{11,12)}$ This implies that the $\mathrm{W}_{2} \mathrm{C}$ phase forms during sintering by the reaction of $\mathrm{W}$ with $\mathrm{WC}$ impurity introduced from the containers and balls made of WC/Co used for MA. ${ }^{11)}$ Although WC/Co exhibits high resistance to erosion during MA, the contam- 
ination of $\mathrm{WC} / \mathrm{Co}$ into $\mathrm{W}-\mathrm{TiC}$ powders is inevitable even by the optimization of MA conditions, i.e., by the optimization of the milling parameters such as the weight ratio of balls to powder, vessel agitation speed, milling time and milling atmosphere. In addition, Co is a highly radioactive element after neutron irradiation in currently available fission reactors such as Japan Materials Testing Reactor (JMTR) and Co contamination must be avoided for post irradiation examinations (PIE). Therefore, we decided to develop new containers and balls made of Mo and its alloy. Because Mo belongs to group VIA, same as $\mathrm{W}$, its carbide, $\mathrm{Mo}_{2} \mathrm{C}$, may be less harmful and has a lower free energy for formantion than $\mathrm{W}_{2} \mathrm{C}$. We also studied the effects of $0.6 \sim 2.4$ mass $\%$ Mo additions on the microstructural evolution, relative density and Vickers microhardness for $\mathrm{W}-0.3$ mass $\% \mathrm{TiC}$, and concluded that the detrimental effect of Mo addition is not significant, which will be reported elsewhere. ${ }^{13)}$ Finally, we have chosen a Mo alloy, TZM (Mo-0.5\% Ti-0.08\%Zr$0.01 \% \mathrm{C}$ in $\operatorname{mass} \%)$, as the material for new containers and balls for MA.

\subsection{Heterogeneity in grain size and particle distribu- tions}

The conditions of MA and the subsequent sintering processes of VHP or HIP are crucial to grain size and particle distributions in sintered compacts. TEM observations of fully MA treated powder of pure $\mathrm{W}$ added with $0.3 \% \mathrm{TiC}$ showed that the grain size of the pure $\mathrm{W}$ phase was as small as $20 \sim 30 \mathrm{~nm}$, and there were no TiC particles. ${ }^{13)}$ This strongly suggests that $\mathrm{TiC}$ was decomposed into $\mathrm{Ti}$ and $\mathrm{C}$ by MA. Such decomposed elements diffuse during VHP or HIP and form dispersoids, which determine the final grain size and its distribution by pinning grain boundaries and suppressing grain growth. Therefore, providing that the starting mixed powders are evenly, fully MA treated, the grain size and particle distributions in sintered compacts are expected to be homogeneous.

However, heterogeneity in grain size and particle distributions was observed in the VHPed or HIPed compacts. This heterogeneity is considered to stem from different degrees of MA from place to place in the container caused by a planetary ball mill: this type of mill tends to enhance adhesion of powder to the inner wall of the container or stagnation of powder agglomerate in the bottom of the container during MA. Adhesion and agglomeration of the powder significantly hinder the homogeneous progress of MA, resulting in heterogeneity in grain size and particle distribution. To produce homogeneously MA treated powders, a 3MPDA (three mutually perpendicular directions agitation) ball mill (Toporogic Systems, Ltd.) with two $250 \mathrm{~mL}$ containers was newly developed. In the MA process using this equipment, the optimization was made so as to meet three requirements: (1) to reach the final stage of MA, (2) to collect a sufficient amount of MA powder, and (3) to suppress contamination with TZM arising from the milling containers and balls during MA. The optimization of the milling parameters requires the determination of the appropriate weight ratio of balls to powder, vessel agitation speed and milling time by trials.

\subsection{Significant loss of carbon}

Significant loss of carbon was confirmed as the result of decarburization during VHP. ${ }^{13)}$ Since the MA treated powder contained an appreciable amount of oxygen as an impurity, $500 \sim 1000$ mass ppm, carbon reacted with the impurity oxygen during VHP above $1720 \mathrm{~K}$ for $1 \mathrm{~h}$ and was removed as $\mathrm{CO}$ gas, of which free energy for formation decreases significantly with increasing temperature. In order to suppress decarburization, the reduction of the oxygen content was attempted. The sintering process was modified so that the consolidated bodies exhibited negligible decarburization and relative densities as high as those produced by VHP ( 99\%). Oxygen reduction was readily achieved by using a specially designed glove box and HIP capsule for consolidation: In the glove box outgassing of the inside wall and all of the necessities including MA vessels and balls, spoons and a balance was accomplished around $420 \mathrm{~K}$ and $10^{-5} \mathrm{~Pa}$ for $10 \mathrm{~h}$ before introducing a purified $\mathrm{Ar}$ or $\mathrm{H}_{2}$ gas (purity 99.9999\%), which provided the milling atmosphere. HIPing with a metal capsule permitted consolidation of the MA treated powder without exposure to the air. First, Ta was selected as the capsule metal. However, HIP using a Ta capsule often led to insufficient densification of the compacts with a relative density between 92 and 97\%, ${ }^{11)}$ which stems from insufficient outgassing from the MA powder and cracking of the capsule during HIP. The sealing of the MA powder within a Ta capsule requires electron beam welding, which is conducted at room temperature in a vacuum and is not suitable for outgassing. Nitrogen or oxygen picked up from the surrounding causes significant hardening and embrittlement of Ta, resulting in difficulty in further densification. Therefore, the authors have proposed the following process to achieve nearly full densification by HIP: At first HIP with a mild steel capsule that allows sufficient outgassing from the MA treated powder is performed at $1620 \mathrm{~K}\left(0.44 T_{\mathrm{m}}\right.$, where $T_{\mathrm{m}}$ is the melting point of $\left.\mathrm{W}\right)$. Then, the sintered compact taken from the metal capsule and wrapped in Ta foil is subjected to further HIP processing at $2220 \mathrm{~K}\left(0.60 T_{\mathrm{m}}\right)$ in an Ar atmosphere.

It should be remarked here that as mentioned later, sintering by HIP with two-step heating at $\sim 0.4$ and $\sim 0.6 T_{\mathrm{m}}$ was found to be very effective to obtain dispersion of nanosize particles of the transition metal carbides, compared with one-step heating at $\sim 0.6 T_{\mathrm{m}} \cdot{ }^{13)}$

\section{Experimetal}

Powders of pure W (an average particle size $4.0 \mu \mathrm{m}$ and purity $99.9 \%)$ and $\mathrm{TiC}(40 \mu \mathrm{m}, 99.9 \%)$ were used as the starting materials. They were mixed to provide a nominal composition of $\mathrm{W}-0.3$ mass $\% \mathrm{TiC}$ and then charged into two vessels made of TZM together with TZM balls for MA. For comparison, containers and balls made of $\mathrm{WC} / \mathrm{Co}$ were also used. MA treatments were conducted by a 3MPDA ball mill under the conditions that the weight ratio of balls to powder was 2, the agitation speed was between 350 and $450 \mathrm{rpm}$, the milling time was between 25 and $50 \mathrm{~h}$ and the milling atmosphere was purifired $\mathrm{Ar}$ or purified $\mathrm{H}_{2}$. The vessels containing the powder and balls were cooled by a fan so that the pot surface temperature was around $280 \mathrm{~K}$. 
Table 1 Specimens, processing conditions, relative densities and the average grain size of the developed alloys by the present method.

\begin{tabular}{ccccccc}
\hline Specimen & Pot \& Ball & $\begin{array}{c}\text { Milling } \\
\text { Atmosphere }\end{array}$ & $\begin{array}{c}\text { Agitation } \\
\text { Speed }(\mathrm{rpm}) / \\
\text { Time }(\mathrm{h})\end{array}$ & $\begin{array}{c}\text { Sintering } \\
\text { Method }\end{array}$ & $\begin{array}{c}\text { Relative } \\
\text { Density } \\
(\%)\end{array}$ & $\begin{array}{c}\text { Average } \\
\text { Grain Size } \\
(\mu \mathrm{m})\end{array}$ \\
\hline W-0.3TiC-0.7Mo & TZM & Purified Ar & $350 / 25$ & 2 step-HIP & 99.5 & 1.14 \\
W-0.3TiC-1.4Mo & TZM & Purified Ar & $350 / 50$ & 2 step-HIP & 99.4 & Forged \\
W-0.3TiC-1.7Mo & TZM & Purified Ar & $450 / 25$ & 2 step-HIP & 99.0 & Forged \& rolled \\
W-0.3TiC-2.2Mo & TZM & Purified H $\mathrm{H}_{2}$ & $350 / 50$ & 2 step-HIP & 99.6 & 2.03 \\
\hline
\end{tabular}

Table 2 Chemical compositions of the developed alloys by the present method together with those processed by the previous method.

\begin{tabular}{|c|c|c|c|c|c|c|c|c|c|}
\hline \multirow{2}{*}{ Specimen } & \multirow{2}{*}{ Pot \& Ball } & \multirow{2}{*}{ MA } & \multirow{2}{*}{$\begin{array}{c}\text { MA } \\
\text { Atmosphere }\end{array}$} & \multirow{2}{*}{$\begin{array}{l}\text { Sintering } \\
\text { Method }\end{array}$} & $\mathrm{C}$ & $\mathrm{N}$ & $\mathrm{O}$ & $\mathrm{Ti}$ & Mo \\
\hline & & & & & \multicolumn{3}{|c|}{ (mass ppm) } & \multicolumn{2}{|c|}{$(\operatorname{mass} \%)$} \\
\hline W-0.3TiC-0.7Mo & $\mathrm{TZM}$ & 3MPDA & Purified Ar & 2 step-HIP & 720 & 80 & 290 & 0.3 & 0.7 \\
\hline W-0.3TiC-1.7Mo & TZM & 3MPDA & Purified Ar & 2 step-HIP & 680 & 50 & 220 & 0.3 & 1.7 \\
\hline $\mathrm{W}-0.3 \mathrm{TiC}-2.2 \mathrm{Mo}$ & $\mathrm{TZM}$ & 3MPDA & Purified $\mathrm{H}_{2}$ & 2 step-HIP & 660 & 40 & 440 & 0.3 & 2.2 \\
\hline W-0.3TiC-1.4Mo & $\mathrm{WC} / \mathrm{Co}$ & $\mathrm{PBM}$ & Purified $\mathrm{H}_{2}$ & 1 step VHP & 120 & 30 & 650 & 0.3 & 1.4 \\
\hline W-0.3TiC-1.4Mo & $\mathrm{WC} / \mathrm{Co}$ & PBM & Purified $\mathrm{H}_{2}$ & 1 step VHP & 50 & 10 & 160 & 0.3 & 1.4 \\
\hline $\mathrm{W}-0.3 \mathrm{TiC}-1.4 \mathrm{Mo}$ & $\mathrm{WC} / \mathrm{Co}$ & PBM & Purified $\mathrm{H}_{2}$ & 1 step VHP & 90 & 10 & 340 & 0.3 & 1.4 \\
\hline
\end{tabular}

The MA treated powder was placed in a Mo boat and heated at $1070 \mathrm{~K}$ for $1 \mathrm{~h}$ in a vacuum to remove the introduced $\mathrm{Ar}$ or hydrogen during MA process. The vacuum-treated powder was charged into a mild steel capsule and then subjected to HIP in an Ar atmosphere at first at $1620 \mathrm{~K}$ and $200 \mathrm{MPa}$ for $3 \mathrm{~h}$ and additional HIP at $2220 \mathrm{~K}$ and $200 \mathrm{MPa}$ for $3 \mathrm{~h}$ for the sintered compact taken from the metal capsule and wrapped in Ta foils. The dimensions of the as-HIPed compacts were approximately $25 \mathrm{~mm}$ in diameter and $26 \mathrm{~mm}$ in height. The HIPed compacts were hot forged and hot rolled around $1770 \mathrm{~K}$ to approximately $1.5 \mathrm{~mm}$ thick. In this case, to avoid abrupt decrease in the specimen temperature during hot working, the as-HIPed compacts were placed in a sheath made of pure $\mathrm{Mo}^{11)}$ prior to hot working.

Table 1 shows the specimens, processing conditions, relative density and grain size of the developed alloys. Table 2 lists the specimens and chemical compositions of the developed alloys by the present method (the upper four alloys) together with those processed by the previous method, i.e., planetary ball milling (PBM) with $\mathrm{WC} / \mathrm{Co}$ containers and balls and sintering with VHP (the lower three alloys ${ }^{13)}$ ) for comparison. Mo was added to $\mathrm{W}-0.3 \mathrm{TiC}$ for the lower three alloys in Table 2, whereas the Mo contents in the upper four alloys came from the vessels and ball of TZM during MA and varied significantly with the MA conditions of agitating speed, milling time and milling atmosphere. This indicates that Mo contamination can be reduced by controlling the MA conditions. It should be noted that the contents of carbon are between 660 and 720 mass ppm as expected from the content of carbon added as $\mathrm{TiC}(0.3$ mass\% TiC). The content of oxygen remains almost constant at a low level, 220 440 mass ppm, whereas it showed considerable variation between the alloys processed by the previous method. ${ }^{11,13)}$ In addition, the measured relative densities of the alloys processed by the present method are 99.0 99.6\%, which is as high as those processed by the previous one, i.e., sintered by VHP. ${ }^{11,13)}$

From the as-HIPed and as-forged compacts, specimens for $\mathrm{X}$-ray diffraction (XRD) analysis, microstructural observations, Vickers and three-point bending tests were prepared. XRD with a voltage of $30 \mathrm{kV}$ and current of $250 \mathrm{~mA}$ was used to identify the dispersed compound particles. Microstructural observations were made by transmission electron microscopy (TEM) with JEM-2000FX and JEM-4000FX operating at 200 or $400 \mathrm{kV}$, respectively. Three-point bending tests were conducted for bend bar specimens with the dimensions of $1 \mathrm{~mm}$ by $1 \mathrm{~mm}$ by $20 \mathrm{~mm}$ using a servo-hydraulic fatigue testing machine (Shimadzu Servopulser of $50-\mathrm{kN}$ capacity equipped with a $5-\mathrm{kN}$ shear-type load cell) with a span of $13.3 \mathrm{~mm}$ and a crosshead speed of $0.013 \mathrm{~mm} / \mathrm{s}$. The crosshead displacements were recorded by a linear variable differential transformer (LVDT) of $\pm 2.5 \mathrm{~mm}$ that was installed on the actuator head.

\section{Results and Discussion}

\subsection{Microstructure}

As shown in Table 2, the content of carbon added as TiC, $\sim 700$ mass ppm, was almost retained in the as-HIPed compacts. Figure 1 shows X-ray diffraction patterns from the as-HIPed (a) W-0.3TiC-0.7Mo and (b) W-0.3TiC-1.7Mo. Definite peaks of $\mathrm{TiC}$ and $\mathrm{Mo}_{2} \mathrm{C}$ without appearance of $\mathrm{W}_{2} \mathrm{C}$ peaks indicate that $\mathrm{TiC}$ and $\mathrm{Mo}_{2} \mathrm{C}$ exist as the dispersed phases.

Figure 2 shows a typical TEM micrograph of a grain structure taken from the as-HIPed W-0.3TiC-1.7Mo. The grain size distribution for $\mathrm{W}-0.3 \mathrm{TiC}-1.7 \mathrm{Mo}$ is shown in Fig. 3, together with that for $\mathrm{W}-0.3 \mathrm{TiC}-1.4 \mathrm{Mo}$ processed by the previous method, i.e., planetary ball milling with WC/Co containers and balls and sintering with VHP at $2120 \mathrm{~K}$. These grain sizes were measured from TEM micrographs. The grain size of $\mathrm{W}-0.3 \mathrm{TiC}-1.7 \mathrm{Mo}$ is in a relatively narrow range from 


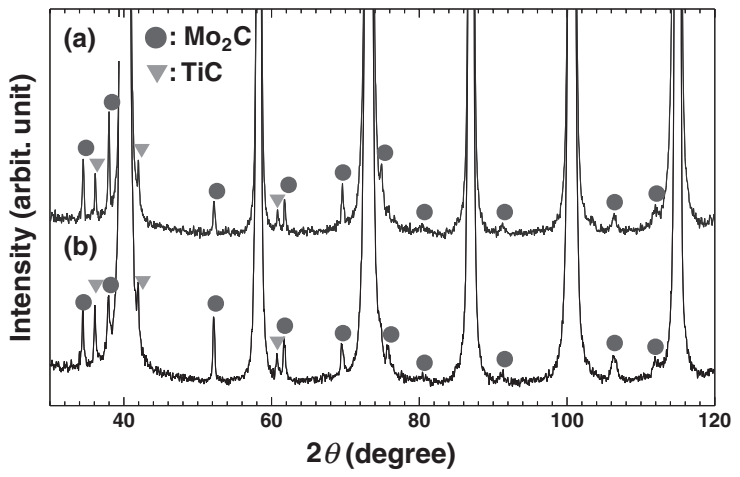

Fig. 1 X-ray diffraction patterns from (a) W-0.3TiC-0.7Mo (TZM) and (b) W-0.3TiC-1.7Mo (TZM) in the as-HIPed state.

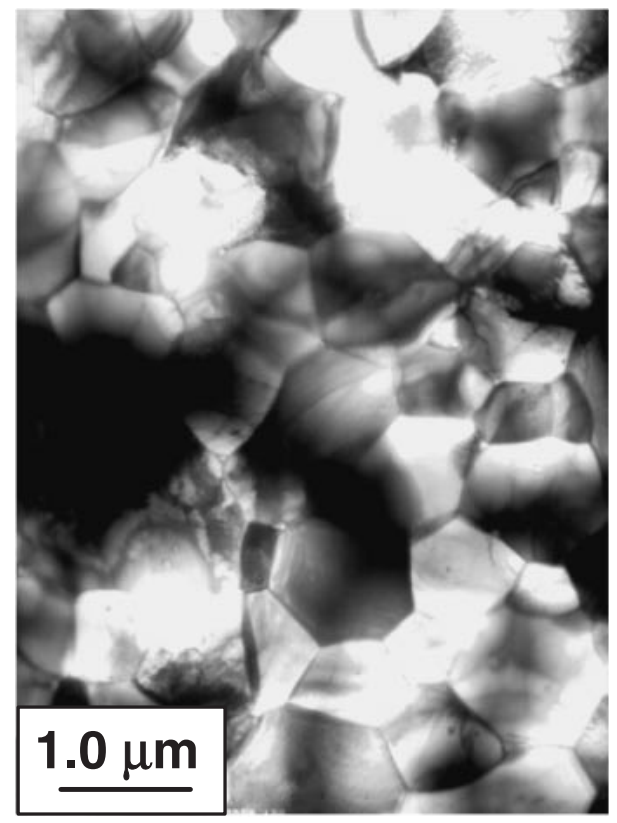

Fig. 2 TEM micrograph showing grain structure of as-HIPed W-0.3TiC1.7Mo (TZM).

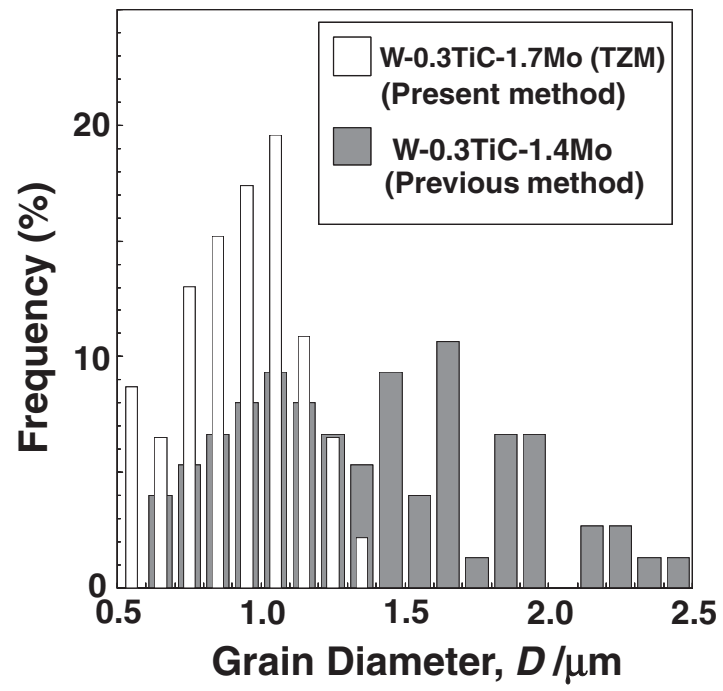

Fig. 3 Grain size distribution for W-0.3TiC-1.7Mo (TZM) developed by the present method together with that for $\mathrm{W}-0.3 \mathrm{TiC}-1.4 \mathrm{Mo}$ processed by the previous method.
0.5 to $1.3 \mu \mathrm{m}$ with an average of $0.91 \mu \mathrm{m}$, whereas that of $\mathrm{W}$ $0.3 \mathrm{TiC}-1.4 \mathrm{Mo}$ is in a wide range from 0.5 to $2.5 \mu \mathrm{m}$ with an average of $1.38 \mu \mathrm{m}$.

Figure 4(a) shows a bright field image of dispersed particles in the as-HIPed W-0.3TiC-1.7Mo. The size distribution of the dispersed particles in the W-0.3TiC-1.7Mo is shown in Fig. 4(b). The particle size is mainly distributed around $10-15 \mathrm{~nm}$ in diameter and particles above $35 \mathrm{~nm}$ are scarce. The average diameter of the particles is $17 \mathrm{~nm}$. On the other hand, for the compact of W-0.3TiC-1.2Mo sintered by VHP at $2120 \mathrm{~K}$ the average particle size was $58 \mathrm{~nm} .{ }^{13)}$ The significant difference in the particle size distribution between $\mathrm{W}-0.3 \mathrm{TiC}-1.7 \mathrm{Mo}$ and $\mathrm{W}-0.3 \mathrm{TiC}-1.2 \mathrm{Mo}$ is attributed to heating history during sintering, because VHP with two-step heating at $1520 \mathrm{~K}$ and $2120 \mathrm{~K}$ for $1 \mathrm{~h}$ leads to a significant decrease in the particle size; from $58 \mathrm{~nm}$ for one-step heating at $2120 \mathrm{~K}$ to $21 \mathrm{~nm}$ for two-step heating. ${ }^{13)}$ This is most likely due to enhanced nucleation and relatively slow growth of the precipitates at $1520 \mathrm{~K}$ compared with the nucleation and growth of the particles in one-step heating at $2120 \mathrm{~K}$ for $1 \mathrm{~h}$. Therefore, it can be stated that sintering with two-step heating at $\sim 0.4$ and $\sim 0.6 T_{\mathrm{m}}$ is very effective to obtain dispersion of nano-size particles of the transition metal carbides.

The average grain size in the four developed alloys is also listed in Table 1 . There is an appreciable difference in grain size between W-0.3TiC-(0.7 1.7)Mo fabricated with a milling atmosphere of Ar gas (grain size: 0.6 1.1 $\mu \mathrm{m}$ ) and W-0.3TiC-2.2Mo with a $\mathrm{H}_{2}$ atmosphere $(2.0 \mu \mathrm{m})$. In order to clarify the cause of the difference, TEM observations were made. Figure 5 shows TEM bright field images of W-0.3TiC$1.7 \mathrm{Mo}$ under the condition of (a) over- and (b) under-focus of the objective lens and (c) W-0.3TiC-2.2Mo. Black and white dots distributed homogeneously in the matrix are observed in Figs. 5(a) and (b), respectively, and these dots are small cavities less than $5 \mathrm{~nm}$ in diameter. The amount of swelling due to the observed cavities was estimated to approximately $0.5 \%$ assuming the specimen thickness of $50 \mathrm{~nm}$. On the other hand, no such small cavities are observed for W0.3TiC-2.2Mo with the milling $\mathrm{H}_{2}$ atmosphere. These observations indicate that the occurrence of cavities is associated with residual Ar gas retained in the HIP capsule and may explain the difference in relative density between W-0.3TiC-1.7Mo (99.0\%) and W-0.3TiC-2.2Mo (99.6\%). It can be hence speculated that the small Ar gas cavities are responsible for the difference in grain size by providing a pinning effect on the grain boundaries during HIP. Milling in hydrogen will likely require more additions for the equivalent degree of grain refinement than milling in Ar.

\subsection{Mechanical properties}

Figure 6 shows three-point bending stress-strain curves for $\mathrm{W}-0.3 \mathrm{TiC}-1.4 \mathrm{Mo}$ in the as-HIPed and as-rolled states and $\mathrm{W}-$ $0.3 \mathrm{TiC}-1.7 \mathrm{Mo}$ in the as-forged state, tested at room temperature and at a displacement rate of $0.013 \mathrm{~mm} / \mathrm{s}$. The stressstrain curves were constructed by converting the Load $(P)$ vs. flexural displacement $(\mathrm{h})$ data to the maximum fiber stress $(\sigma)$ and strain $(\varepsilon)$ at the center of the specimen. The following well-known relations are given in three point bending. 

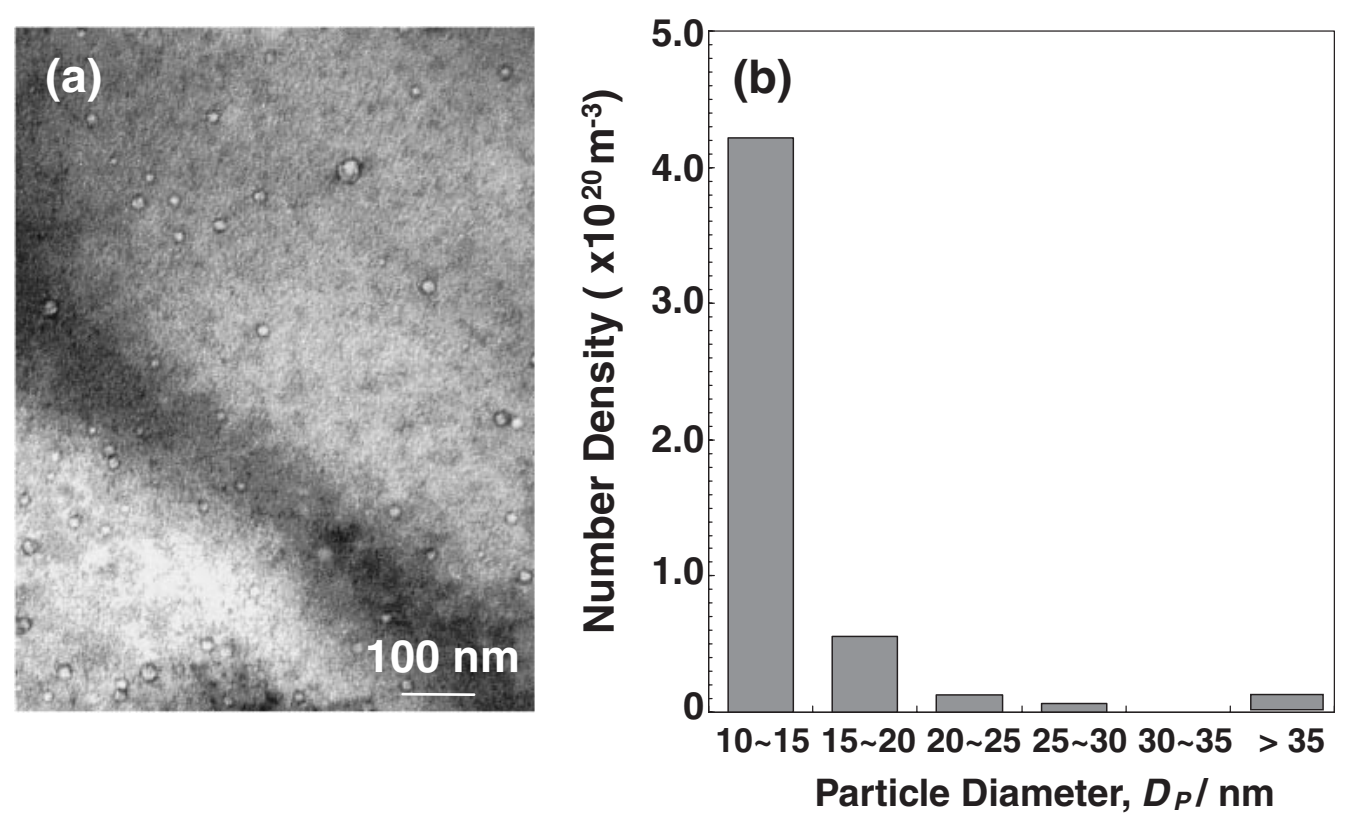

Fig. 4 TEM micrographs showing (a) dispersed particles and (b) its size distribution in the as-HIPed W-0.3TiC-1.7Mo (TZM).
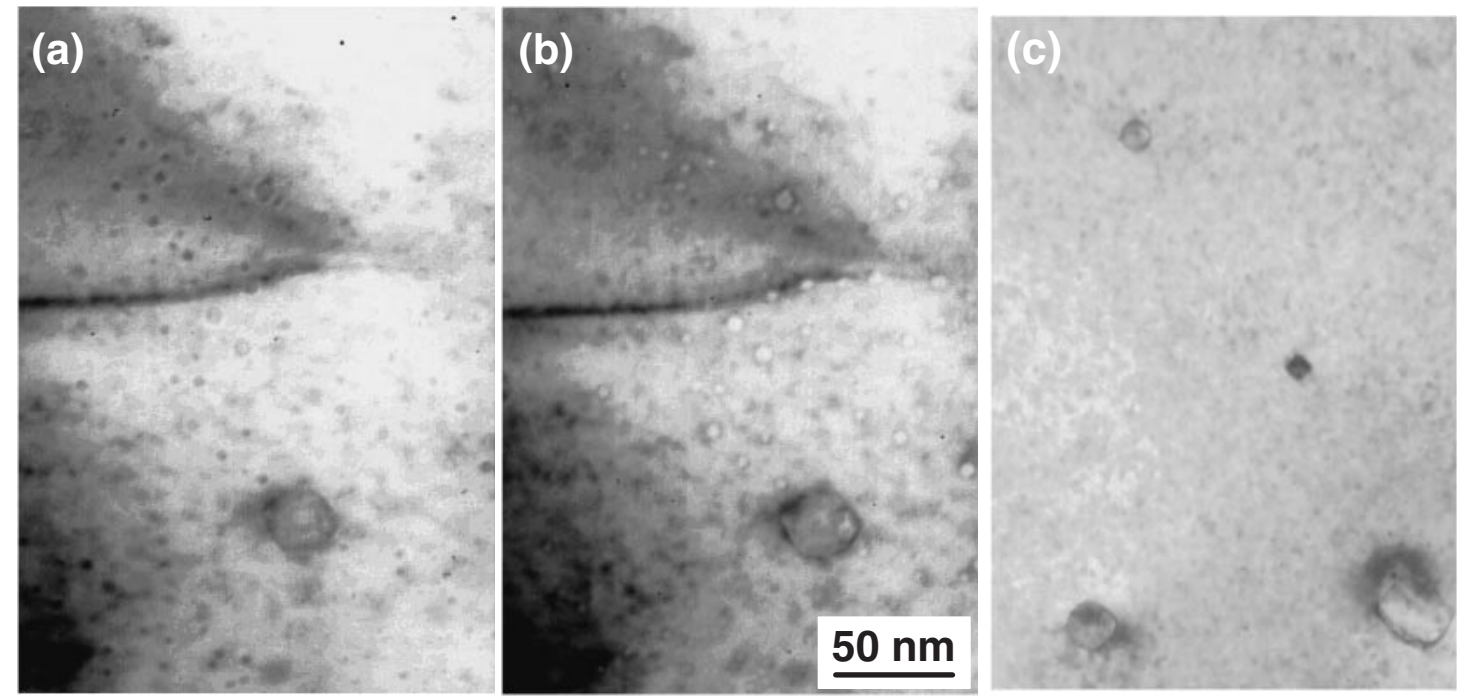

Fig. 5 TEM microgrphs of as-HIPed W-0.3TiC-1.7Mo (TZM) fabricated with an Ar atmosphere for milling under (a) over- and (b) underfocus of objective lens and (c) as-HIPed W-0.3TiC-2.2Mo (TZM) with a $\mathrm{H}_{2}$ atmosphere for milling.

$$
\sigma=3 P L / 2 w t^{2}, \quad \varepsilon=6 t h / L^{2} .
$$

Here, $L$ is the span $(13.3 \mathrm{~mm}), w$ and $t$ are the specimen width and thickness, respectively. Eq. (1) holds in the elastic region, whereas the values of $\sigma$ and $\varepsilon$ begin to deviate from eq. (1) after yielding. However, the observed plastic deformation prior to fracture is not large. The obtained stress-strain curves are hence valid enough to compare the mechanical behavior of the alloys shown in Fig. 6. It is obvious from Fig. 6 that in the as-HIPed state brittle fracture occurs without showing any ductility, whereas appreciable plastic deformation precedes fracture in the as-forged and as-rolled states. Plastic working after HIP hence significantly improves the room temperature ductility. This effect of plastic working for fine- grained and particle-dispersed tungsten developed by MAHIP is not clear yet, but possible explanations are: (1) the plastic working causes elongated and very thin grain structures that are beneficial in increasing ductility owing to increase in a delamination-like effect and decrease in the effective size of weak gain boundaries acting as crack initiators and (2) the residual cavities and pores can be eliminated from the matrix by interaction with a high density of dislocations introduced during plastic working.

As mentioned above, the appreciable difference in the average grain size was recognized between the developed alloys. Therefore, the bending fracture strength was plotted against $D^{-1 / 2}$, where $D$ is the average grain size, for the developed alloys in the as-HIPed, as-forged and as-rolled 


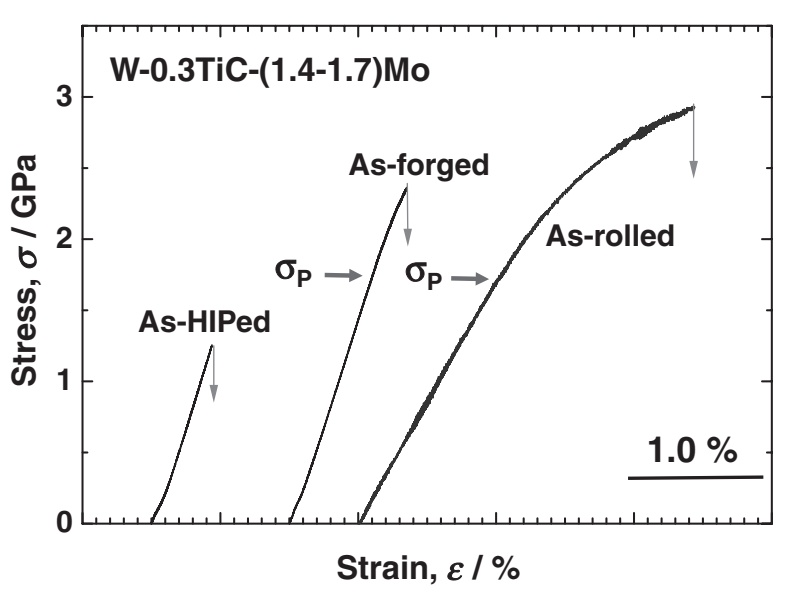

Fig. 6 Stress-strain curves by three-point bending at room temperature and at a cross-head speed of $0.013 \mathrm{~mm} / \mathrm{s}$ for the as-HIPed, as-forged and asrolled W-0.3TiC-(1.4 1.7)Mo (TZM), showing that plastic working after consolidation significantly improves the ductility.

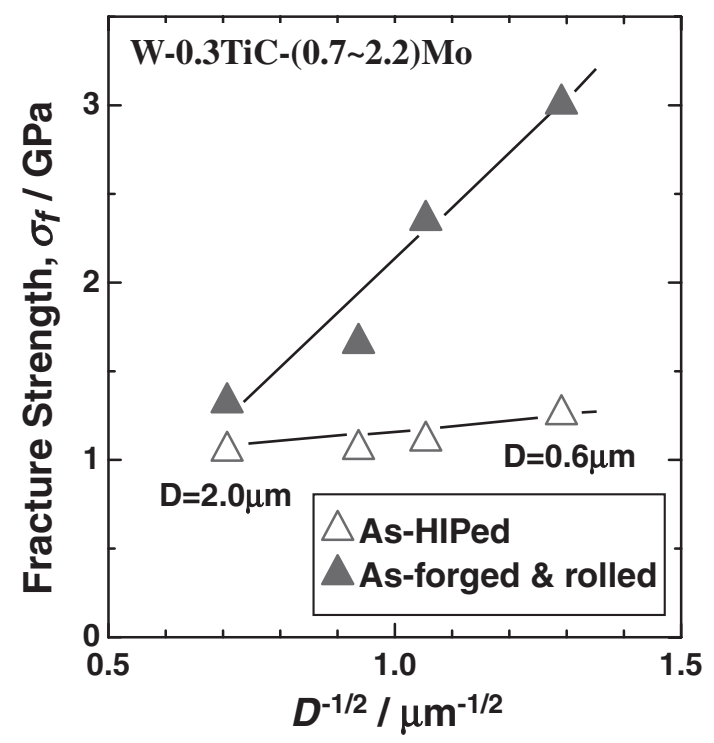

Fig. 7 Fracture strength against the reciprocal of average grain diameter for $\mathrm{W}-0.3 \mathrm{TiC}-(0.7 \sim 2.2) \mathrm{Mo}$ (TZM) in the as-HIPed, as-forged and asrolled states.

states. The result is shown in Fig. 7. In both cases the fracture strength increases with decreasing grain size, showing an approximate linear relationship with $D^{-1 / 2}$. It should be noted that the slope of the linear relationship is much higher for the as-forged and as-rolled specimens than for the asHIPed specimens. It can be expected that the beneficial effect of plastic working after consolidation on ductility improvement becomes prominent with decreasing grain size. This suggests that more marked improvement in ductility will be achieved by fabricating consolidated bodies with very fine grains less than $0.6 \mu \mathrm{m}$ in diameter and giving a sufficient degree of plastic working.

In the previous paper, the authors showed that fabrication of compacts with such ultra-fine grains can be made by HIPing MA powders at lower temperatures to suppress grain growth. ${ }^{6,9)}$ However, as the HIPing temperature was lowered, the relative density of the resultant as-HIPed compacts

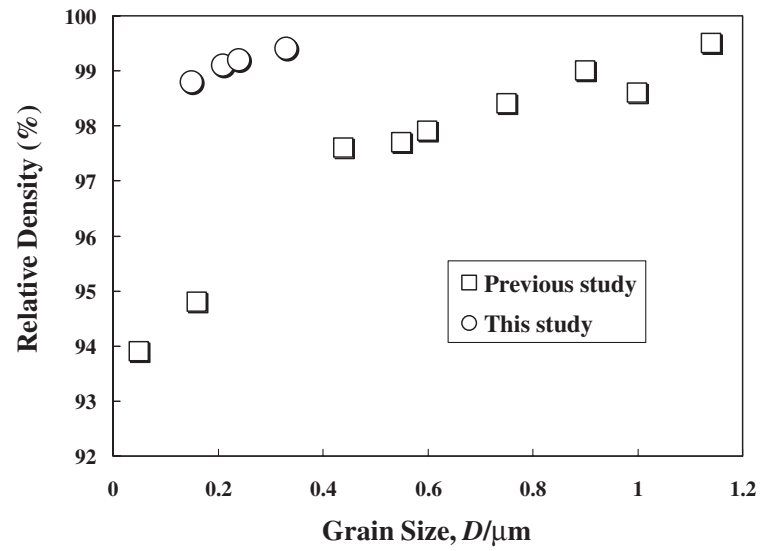

Fig. 8 Relationship between the relative density and grain size for the assintered compacts of $\mathrm{W}-(0.3-0.5) \% \mathrm{TiC}$.

decreased; e.g., the relative density and the grain size of a compact HIPed at $1620 \mathrm{~K}$ for $5 \mathrm{hr}$ were $93.9 \%$ and approximately $50 \mathrm{~nm}$, respectively. ${ }^{6,9)}$ Therefore, fabrication methods of consolidated bodies have been attempted to be modified in order to achieve grain sizes less than $0.6 \mu \mathrm{m}$ and relative density of around $99 \%$. The key issue was to develop a method that allows sufficient outgassing from the MA treated powder during encapsulation of the powders in a metal can before HIP. As a result, the authors have succeeded in fabricating HIPed compacts of W-(0.3-0.5)\% TiC-(2.0 $2.4) \%$ Mo with extremely fine grains of $0.17 \sim 0.4 \mu \mathrm{m}$ and relative density of $98.8 \sim 99.4 \%$, as shown in Fig. 8. This achievement in fabrication of $\mathrm{W}$ alloys with extremely fine grains and high densities is the first in $\mathrm{W}$ and its alloys and should be noteworthy. Plastic working for these HIPed compacts is now in progress.

\section{Conclusions}

To eliminate the detrimental effects of three microstructural factors on the ductility and recrystallization temperature, such as (1) precipitation of the brittle $\mathrm{W}_{2} \mathrm{C}$ phase, (2) heterogeneity in grain size and particle distribution and (3) significant loss of carbon which is a constituent of finely dispersed particles of transition metal carbides, an improved process was proposed and applied to fabricate $\mathrm{W}-0.3 \% \mathrm{TiC}$ alloys. The alloys were subjected to microstructural observations, XRD analysis and three-point bending tests at room temperature. The main results are as follows.

1) The developed alloys exhibit grain sizes typically ranging from 0.5 to $1.3 \mu \mathrm{m}$ and particle sizes with a sharp distribution around $10-15 \mathrm{~nm}$, featuring homogeneous distributions in grain size and particle size. Such fine dispersion of the particles was achieved by two-step heating at $\sim 0.4$ and $\sim 0.6 T_{\mathrm{m}}$ for HIP.

2) $\mathrm{XRD}$ analysis showed peaks of $\mathrm{TiC}$ and $\mathrm{Mo}_{2} \mathrm{C}$, indicating that the dispersed particles are $\mathrm{TiC}$ and $\mathrm{Mo}_{2} \mathrm{C}$. Mo was introduced from the vessels and balls of TZM during MA.

3) The developed alloys retain almost the same carbon content as that added as TiC. The oxygen contents in the alloys were low, 220 440 mass ppm. 
4) Appreciable difference in grain size was recognized between the alloys fabricated with a milling atmosphere of Ar gas and those with a $\mathrm{H}_{2}$ atmosphere, the former being finer. The finer grains in the former alloys are attributable to very small cavities, less than $10 \mathrm{~nm}$, that are associated with residual Ar gas and provide the effects of pinning grain boundaries and inhibiting grain growth during the HIPing.

5) The developed alloys exhibit appreciable room-temperature ductility before fracture in the as-forged and as-rolled states, but not in the as-HIPed state. This beneficial effect of plastic working on ductility improvement strongly depends on grain size and becomes prominent with decreasing grain size.

6) Consolidated bodies with grain sizes as small as $0.17 \sim$ $0.4 \mu \mathrm{m}$ and a high relative density of around $99 \%$ were successfully fabricated.

\section{Acknowledgments}

The authors would like to express their gratitude to Dr. S. Matsuo for his review. The present work was supported by Grant-in-Aid for Scientific Research (A) (\#13308022), Japan Society for the Promotion of Science (JSPS), and by NIFS Collaborative Research Program, which are greatly appreciated.

\section{REFERENCES}

1) For instance, I. Smid, M. Akiba, G. Vieider and L. Plöchl: J. Nucl. Mater. 258-263 (1998) 160-172.

2) J. S. Benjamin: Metall. Trans. 5 (1970) 2943.

3) H. Kurishita, Y. Kitsunai, Y. Hiraoka, T. Shibayama and H. Kayano: Mater. Trans., JIM. 37 (1996) 89-97.

4) Y. Kitsunai, H. Kurishita, T. Shibayama, M. Narui, H. Kayano and Y. Hiraoka: J. Nucl. Mater. 239 (1996) 253-260.

5) K. Tokunaga, Y. Miura, N. Yoshida, T. Muroga, H. Kurishita, Y. Kitsunai and H. Kayano: J. Nucl. Mater. 241-243 (1997) 1197-1202.

6) H. Kurishita, Y. Kitsunai, T. Kuwabara, M. Hasegawa, Y. Hiraoka, T. Takida and T. Igarashi: J. Plasma and Fusion Res. 75 (1999) 594603 (in Japanese).

7) H. Kurishita: Basic Studies in the Field of High-Temperature Engineering, OECD, (2002) 103-112.

8) T. Takida, H. Kurishita, M. Mabuchi, T. Igarashi, Y. Doi and T. Nagae: Mater. Trans. 45 (2004) 143-148.

9) Y. Kitsunai, H. Kurishita, M. Narui, H. Kayano, Y. Hiraoka, T. Igarashi and T. Takida: J. Nucl. Mater. 271-272 (1999) 423-428.

10) Y. Ishijima, H. Kurishita, K. Yubuta, H. Arakawa, M. Hasegawa, Y. Hiraoka, T. Takida and K. Takebe: J. Nucl. Mater. 329-333 (2004) 775-779.

11) Y. Ishijima, H. Kurishita, M. Hasegawa, Y. Hiraoka, T. Takida and K. Okamoto: submitted to J. Nucl. Mater.

12) S. Kannari, Y. Ishijima, H. Kurishita, K. Yubuta, M. Hasegawa, Y. Hiraoka, T. Takida and K. Okamoto: in preparation.

13) Y. Ishijima, H. Kurishita, K. Yubuta, M. Hasegawa, Y. Hiraoka, T. Takida and K. Okamoto: in preparation. 